\title{
Visual representation in analogical problem solving
}

\author{
M. BEVERIDGE and E. PARKINS \\ University of Manchester, Manchester, England
}

\begin{abstract}
Analogical reasoning has been shown to be effective in the process of solving Dunker's radiation problem. The spatial nature of the solution to this problem suggests that a visually represented analogue should be particularly effective. However, recent work seems to indicate that a visual analogue does not assist in solving the radiation problem. This paper reports a detailed experimental analysis of the effectiveness of visually represented analogues to the radiation problem. The results show that visual analogues can be effective if they represent the appropriate features of the problem-solution relationship. The paper also reports on the use of an appropriate visual representation within the problem as a facilitator of analogical reasoning. The results indicate that a visual representation within the problem can act as a facilitator of analogical reasoning, possibly by acting as a retrieval cue.
\end{abstract}

Previous work on problem solving by analogy has indicated that analogues may be presented effectively in either a verbal form (Reed, Ernst, \& Banjeri, 1947) or a visual form, such as pictures or diagrams (Driestadt, 1969). Furthermore, it has been suggested that, whatever the form of presentation, analogical reasoning often involves visual mental representation, especially when applied to spatial problems, as in Driestadt (1969) (see Beck, 1978; Chafe, 1976; Kintsch, 1974; Kosslyn, 1975; Kosslyn \& Pomerantz, 1977; Norman \& Rumelhart, 1975; Palmer, 1976; Shepard, 1975).

Of particular interest, therefore, is the work of Gick and Holyoak $(1980,1983)$ on the use of visual representation, by diagrams, in problem solving. Gick and Holyoak reported a series of experiments that failed to show a significant effect of presenting visual cues in a problem-solving context. Although Gick and Holyoak investigated a number of problems for solution by analogy, the one on which their conclusions about visual representation were based was the radiation problem described by Duncker (1945). Duncker's radiation problem as used by Gick and Holyoak is stated as follows:

Suppose you are a doctor faced with a patient who has a malignant tumor in his stomach. It is impossible to operate on the patient, but unless the tumor is destroyed the patient will die. There is a kind of ray that can be used to destroy the tumor. If the rays reach it all at once at a sufficiently high intensity, the tumor will be destroyed. Unfortunately, at this intensity the healthy tissue that the rays pass through on the way to the tumor will also be destroyed. At lower intensities the rays are harmless to healthy tissue, but they will not affect the tumor either. What type of procedure might be used to destroy the tumor with the rays, and at the same time avoid destroying the healthy tissue?

Please address all correspondence to the first author at: Department of Education, University of Manchester, Oxford Road, Manchester M13 9PL, England.
One of the possible solutions identified by Dunker (1945) is to reduce the intensity of the rays as they pass through the healthy tissue. In this solution, the tumor is destroyed by sending several weak $\mathrm{x}$-rays from different directions so that they converge, in effect producing intense $x$-rays in the tumor. Gick and Holyoak $(1980,1983)$ constructed a number of verbal propositional (story) analogues to this dispersion solution for the radiation problem. One of the stories used in their work was "Red Adair."

An oil well in Saudi Arabia exploded and caught fire. The result was a blazing inferno that consumed an enormous quantity of oil each day. After initial efforts to extinguish it failed, famed firefighter Red Adair was called in. Red knew that the fire could be put out if a huge amount of fire retardant foam could be dumped on the base of the well. There was enough foam available at the site to do the job. However, there was no hose large enough to put all the foam on the fire fast enough. The small hoses that were available could not shoot the foam quickly enough to do any good. It looked like there would have to be costly delay before a serious attempt could be made.

However, Red Adair knew just what to do. He stationed men in a circle all around the fire, with all of the available small hoses. When everyone was ready all of the hoses were opened up and foam was directed at the fire from all directions. In this way a huge amount of foam quickly struck the source of the fire. The blaze was extinguished, and the Saudis were satisfied that Red had earned his three million dollar fee.

In the initial experiments, Gick and Holyoak (1980) presented the stories only in verbal propositional form. After having read the story, the subjects immediately were asked to attempt the radiation problem without a prior hint to use the story. Then they were asked to propose a solution suggested by the story.

Although the stories were presented verbally in Gick and Holyoak's (1980) experiments, many of the subjects made comments that suggested that visuospatial images 
representing the essential aspects of the dispersion solution were useful in solving the radiation problem. This led Gick and Holyoak (1983) to investigate whether a visuospatial representation of the solution in the form of a diagram could itself serve as an analogue for the problem. They also looked at whether such a diagram made a significant improvement to the utilization of a story analogue when story and diagram were simultaneously presented. Figure 1 shows the diagram used.

When the diagram alone was presented, it was introduced as a pattern-recognition memory task. Gick and Holyoak (1983) found that after the diagram had been presented alone, before the radiation problem, only $7 \%$ of the subjects solved the problem; after a hint, $60 \%$ solved the problem, but this was less effective than the story analogue alone. Moreover, Gick and Holyoak found that the diagram did not make a significant improvement in the utilization of the story. In fact, in the prehint condition, the story-plus-diagram group achieved $23 \%$ success, whereas the story-alone group achieved $40 \%$ success. Gick and Holyoak's report therefore suggests that, despite the visuospatial nature of this particular problem, a visually presented representation of the solution, in the form of a diagram, does not facilitate analogical problem solving. This result conflicts directly with the work of Driestadt (1969), which indicated that pictures or diagrams could serve as valid analogues even without hints.

Gick and Holyoak (1983) explained the relative ineffectiveness of their diagrammatic representations by suggesting that the diagram did not mean anything to the subjects initially. They also suggested that, when presented along with the story analogue, the diagram was interpreted concretely as "roads" or "hoses": That is, the diagram was not seen as representations of a general solution schema that could be mapped onto the problem. The diagram therefore failed to facilitate analogical transfer from the story analogue to the $\mathrm{x}$-ray problem.

However, a more fundamental and empirically testable reason why Gick and Holyoak (1983) failed to obtain a spontaneous effect from their visual cues could be that the diagram they used was inadequate as a representation of the problem-solution schema required to solve the $\mathrm{x}$ ray problem. The dispersion solution of the $\mathrm{x}$-ray problem has two essential features. One concerns a change of spatial arrangement (instead of a single beam sent in one

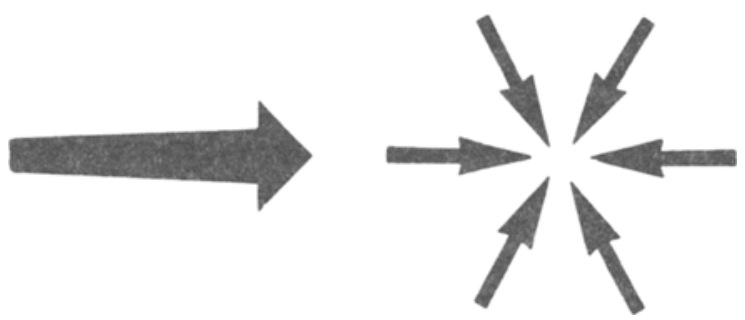

Figure 1. Diagram representing the dispersion solution used by Gick and Holyoak (1983).

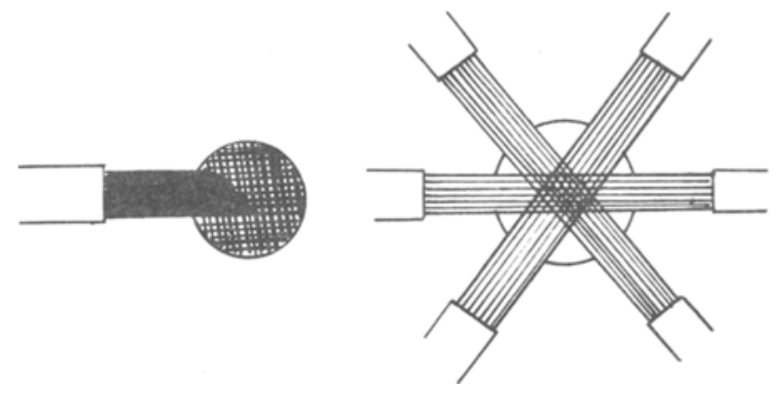

Figure 2. Diagram representing the dispersion solution in our experiments.

direction, use several from different directions), and the other concerns a change of intensity that includes a summative effect (instead of one strong beam, use several weak beams which summate at the point of convergence).

The diagram Gick and Holyoak (1983) used (see Figure 1) represented the dispersion solution by a series of converging arrows to indicate different forces acting at a point. This representation is consistent with the spatial arrangement of the solution to the problem, but does not unequivocally indicate intensity, since both the large and the small arrows are equally dark in tone. Moreover, the diagram does not indicate any summative effect, which is a crucial aspect of the solution.

One of the objectives of our research, then, was to determine whether or not visual analogues could be effective in solving the $x$-ray problem if they explicitly represented intensity and summation at the point of convergence. Figure 2 shows the visuospatial analogue of the $x$-ray problem used here. Figure 2 uses differences in shading to explicitly represent the difference in intensity at the intersection of the radials. The summative effect of the x-rays can be mapped directly onto this "hub" of the diagram.

However, diagrams are not the only visual form that can represent changes in intensity and spatial arrangement. It would be inappropriate, therefore, to conclude from failure of diagrammatic analogues that visual analogues are ineffective in problem solving, especially because utilizing information from diagrams requires an understanding of their conventions (e.g., shading indicates in tensity). Furthermore, diagrams differ in "eye-catching"' quality, memorizability, and complexity (Willows, Borwick, \& Hayvren, 1981), all of which may influence their effectiveness as visual analogues.

The nondiagrammatic verbal analogue used here took the form of a colored-strips display (see Figure 3 ) in which transparent blue plastic strips, hinged together at one end, were slowly fanned out to reveal the change from dark to light shades of blue on the nonoverlapping parts of the strip. This visually presented "real" event maintains the explicit representation of the summative effect of intensity at the center of the display. This colored-strips display was also thought to be attended to easily and as- 

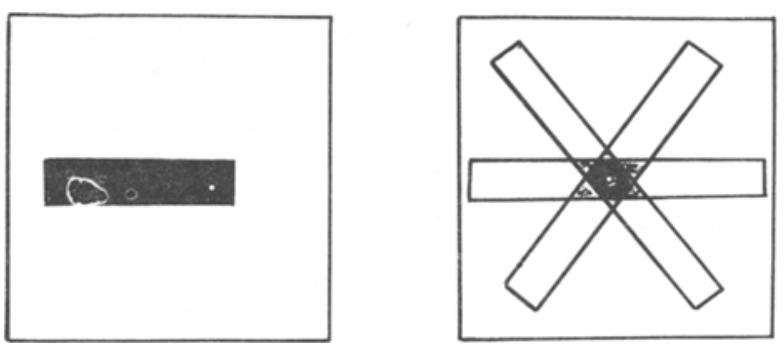

Figure 3. The colored strips before (left) and after (right) being fanned out.

similated through its eye-catching nature. Experiments 1 and 2 reported here investigated the effects of both types of visual analogue, diagrams and colored strips.

The second objective of the research reported here was to determine whether a visual representation can act as an effective retrieval cue. Analogical reasoning in problem solving depends in part on recall of the analogue from memory (Gick \& Holyoak, 1980). Gick and Holyoak suggested that it may be possible to use a representation within the problem as a retrieval cue for accessing its analogue. Experiments 3 and 4 reported here presented a story analogue with the diagrams in Figure 2 and investigated the effect of presenting the problem either with or without the diagram that represents the problem.

The subjects in Experiments 1 and 3 were children; those in Experiments 2 and 4 were adults. Holyoak, Junn, and Billman (1984) had identified analogical problemsolving skills in children, and the present experiments extended this work to see whether children could also take advantage of the more appropriate visual analogues described earlier.

\section{EXPERIMENT 1}

To overcome the inadequacies of Gick and Holyoak's (1983) visual analogue, Experiment 1 examined the effectiveness of a visual analogue that included the three features of (1) intensity difference, (2) summation at the focus, and (3) change in intensity. A control group of subjects was compared with a group receiving the coloredstrips analogue and a group receiving the verbal Red Adair analogue.

\section{Method}

\section{Materials}

X-ray problem. Duncker's $x$-ray problem was modified in two ways to adapt it to the age range being studied. First, the description of the problem itself was simplified. Second, an introductory paragraph was included to give the necessary information about the properties of $\mathrm{x}$-rays. As a result of this second modification, it could safely be assumed that all the pupils able to read the problem would also have the relevant information about $x$-rays. This modified version of the $x$-ray problem is presented below.

\section{$X$-Ray Problem}

$A$ beam of $x$-rays is a bit like a beam of light from a torch, but $x$-rays can travel through the body. A large amount of $x$-rays will destroy the body, but a small amount will not. A large amount of $x$-rays can be sent through a part of the body by pointing a strong $\mathrm{x}$-ray lamp through it or by pointing a number of weak $\mathrm{x}$-ray lamps through it at the same time-just as a large amount of light can be sent onto a part of a wall by pointing a strong torch onto it or by pointing a number of weak torches onto it at the same time.

\section{Doctor's Problem}

A patient had got a 'bad growth' deep inside his body. The patient was very old and knew that he could not be cut open to have the bad growth removed. He went along to his doctor to see what could be done. The doctor knew what to do to get rid of the bad growth. He could destory the bad growth by sending a large amount of $x$-rays through it. A way in which he could do this would be by pointing one strong beam of $x$-rays through the bad growth. Unfortunately the doctor could not use a strong beam of $x$-rays because it would destroy the good part of the body as it passed through on the way to the bad growth. The doctor knew that he could send a weak beam of $x$-rays through the body and this would not destroy the good part of the body, but it would not destroy the bad growth either!

\section{Question}

How could the doctor send a large amount of $x$-rays through the bad growth to destroy it without destroying the good part that surrounds it? If you think of more than one way to do this, give more than one answer.

Red Adair analogue. The Red Adair analogue used by Gick and Holyoak (1980) and described above was modified for the age range studied. It was also verbally matched to the modified $x$-ray problem according to the procedure of Gick and Holyoak (1980). This modified version of the analogue is presented below.

An oil well in Saudi Arabia exploded and caught fire. The workmen at the well could not manage to put the fire out and so they called in Red Adair, the famous fire fighter. Red knew what to do to get rid of the fire. He could put out the fire by quickly sending a large amount of extinguisher foam onto it. A way in which he could do this would be by using a wide hose to point one strong jet of foam onto the fire. Unfortunately Red could not get a wide enough hose-pipe to do this. All that Red could get was a number of narrow hoses, but each one of these on its own could only send out a weak jet of foam and this was not enough to put out the fire. Suddenly Red had an idea. He got a number of workmen each to take one narrow hose and then to stand in a circle round the fire. The workmen got into position pointing their hoses toward the fire at the center of the circle; then all the hoses were turned on at once. Each narrow hose sent out a weak jet of foam, but at the center of the circle where the jets came together there was enough foam to put out the fire.

Recall questions for Red Adair analogue. The following three recall questions about the Red Adair analogue were asked before the Red Adair subjects attempted to solve the $x$-ray problem.

1. Could a wide hose-pipe send a large amount of foam quickly onto the fire to put it out?

2. Could a narrow hose-pipe send a large amount of foam quickly onto the fire to put it out?

3. Red Adair only had narrow hose-pipes. How did he arrange these to get a large amount of foam quickly onto the fire?

Colored-strips analogue. In the colored-strips analogue, the in formation analogous to the $\mathrm{x}$-ray problem solution is represented in a visual, nonverbal form. For this analogue, six thin transparent blue plastic strips $20 \mathrm{~cm}$ long $\times 3 \mathrm{~cm}$ wide were placed in a pile on top of one another as shown in Figure 3. The strips were attached near one end to the center of a white $50 \times 50 \mathrm{~cm}$ card. In this position, the pile of blue strips appeared dark blue.

After the children had observed the colored strips in their initial arrangement, the strips were moved into the position shown in 
Figure 3. In this arrangement, the colored strips appeared to be strong (dark) blue where they overlapped in the center, whereas they appeared to be weak (light) blue where they did not overlap.

Recall questions for colored-strips analogue. The following recall questions about the colored-strips analogue were asked before the subjects attempted to solve the $\mathrm{x}$-ray problem.

1. Did the thick pile of blue strips look a strong blue or a weak blue?

2. Did a single thin blue strip look a strong blue or weak blue?

3. How were the blue strips arranged, so that part of them looked weak blue and part of them strong blue?

\section{Design and Procedure}

Three hundred 10-11-year-old pupils from 12 schools participated in Experiment 1. The subjects were tested on the same day to prevent contamination of the groups. The experiment was carried out in each class by the usual class teacher according to a set procedure. The two analogues were presented to the children immediately before the morning playtime break. These analogues were presented to the class as memory tasks; the children had to try to remember certain features of the analogue (in particular those features relevant to the $\mathrm{x}$-ray problem). The Red Adair analogue was presented in written form to each child individually. The coloredstrips display was presented to the class as a whole by demonstration. Teachers were instructed not to give any hints. After the students had written their answers, the teacher collected the answers. The children were allocated randomly by school to one of three experimental groups. The control group received only the $\mathrm{x}$-ray problem, which they attempted to solve without receiving an analogue beforehand. This group comprised 29 pupils from two schools. The Red Adair story group first received the Red Adair story analogue and then, after a break, attempted (1) to answer the analogue recall questions and (2) to solve the X-ray problem. This group comprised 106 pupils from four schools. The colored-strips group first received the colored-strips analogue presented to each school class in a single demonstration, and then, after a break, attempted (1) to answer the analogue recall questions, and (2) to solve the $x$-ray problem. The group comprised 165 pupils from six schools.

For the children in the Red Adair and colored-strips groups, scores on a nonverbal reasoning test consisting of 25 multiple-choice questions in each of four subtests (Cypher, Similarities, Analogies, and Series) were obtained. An analysis of variance of the nonverbal reasoning test for those subjects whose $\mathrm{x}$-ray problem answers were considered in the final assessment showed that the two analogue groups were equivalent in terms of their overall scores and analogues sections scores.

The analogue recall questions provided the children with a reminder of the analogue and therefore served as an implicit hint. They also indicated which pupils could actually remember the essential features of the analogue. It was assumed that those children who could not correctly answer all three recall questions could not remember the essential features of the analogue and thus could not use the analogue to solve the problem. The $x$-ray problem answers of those children who did not correctly answer all three recall questions were not considered in the final assessment of the results. (In fact, none of the children answered the $x$-ray problem correctly unless they had also answered the three recall questions correctly.) Ignoring these answers ensured that all the analogue groups were equivalent in that all the pupils within each of the groups had the essential features of the analogue within memory and that differences between the final results of the groups therefore could not be attributed to differential memory effects. This procedure also meant that the evaluation of the relative effectiveness of the Red Adair and colored-strips analogues would not reflect differences in the ease of recall of the two types of analogue; rather, it would reflect differences in the ease of recognition of the analogical relationship between the analogue and the problem.

\section{Assessment of X-Ray Problem Answers}

The answers to the $\mathrm{x}$-ray problem were judged simply as correct or incorrect according to whether or not the answer contained, explicitly or implicitly, the following three features: (1) A number of weak x-rays that were (2) applied from different directions and that (3) came together in the bad growth. This is basically the same evaluative procedure employed by Gick and Holyoak (1980). Judgments were blind with respect to experimental condition, and independent reliability checks revealed no cases of disagreement.

\section{Results}

Table 1 shows that none of the subjects in the control group solved the $x$-ray problem, whereas $14 \%$ of the Red Adair group and $32 \%$ of the colored-strips group were successful. The difference between these last two conditions was significant $\left(\chi^{2}=7.8, p<.001\right.$, one-tailed). The difference between the Red Adair group and the control group was also significant $\left(\chi^{2}=5.2, p<.01\right.$, onetailed). These results show a positive effect of a visual analogue that is a more adequate representation of the problem than was the analogue used by Gick and Holyoak (1983). This calls into question their conclusion that visual analogues do not facilitate problem solving.

\section{EXPERIMENT 2}

There is an important difference between Gick and Holyoak's (1983) presentation of the problem and that in Experiment 1: An introductory paragraph was added to ensure that the children knew enough relevant information about $\mathrm{x}$-rays. This paragraph, although on its own not giving enough of a hint for the children to solve the problem, could have been interacting with the analogues to enhance their apparent positive effect. Therefore, Experiment 2 was carried out with the unmodified problem and with adult subjects to provide a more direct comparison with Gick and Holyoak (1983). It also investigated the conclusion of Holyoak et al. (1984) that analogical reasoning is essentially the same process in adolescents and adults. The $\mathrm{x}$-ray problem used in this experiment was that used by Gick and Holyoak (1983) and presented earlier in the introduction. It did not include a diagram.

Table 1

Number and Percentage of Correct Solutions to the Problem for Each Analogue Type in Experiments 1 and 2

\begin{tabular}{lcc} 
& & \multicolumn{2}{c}{ Correct Solutions } \\
\cline { 3 - 3 } Analogue Type & Number & Percentage \\
\hline & Experiment 1 & (Children) \\
Control & $0 / 29$ & 0 \\
Red Adair & $70 / 71$ & 14 \\
Colored Strips & $38 / 118$ & 32 \\
& & \\
Control & Experiment 2 (Adults) & \\
Red Adair & $24 / 40$ & 57 \\
Gick and Holyoaks (1983) & $30 / 40$ & 75 \\
Diagram & & \\
Summative Intensity & $28 / 40$ & 63 \\
Colored Strips & $44 / 55$ & 82 \\
\hline
\end{tabular}


A further question was also addressed in this experiment. It has already been noted that Gick and Holyoak's (1983) diagram lacked explicit visual representation of the summative intensity of the converging $x$-rays. It may be that diagrams that do contain such a representation would also facilitate solving the problem. Such diagrams were produced (see Figure 2), and their effect is contrasted here with the effect of the diagram used by Gick and Holyoak (1983).

\section{Method}

A total of 215 university students participated in this experiment. Those groups receiving analogues received them immediately before attempting to solve the $\mathrm{x}$-ray problem. No recall questions were asked before or after the students attempted the problem. No hint to use the analogue in solving the problem was given. Each analogue, except the colored-strips analogue, was presented on a printed sheet to each student individually. The colored-strips display was presented to groups of approximately 10 students at a time, by demonstration. The answers to the problem were assessed in the same way as in Experiment 1 . All subjects received the unmodified x-ray problem, as used by Gick and Holyoak (1983). A control group of 40 subjects received only the problem. Prior to receiving the problem, the other groups were assigned randomly to the following experimental conditions. Forty subjects received the colored-strips analogue, 55 subjects received the diagrams representing summative intensity, 40 subjects received the Gick and Holyoak (1983) diagram, and 40 subjects received the unmodified Red Adair analogue, in Gick and Holyoak (1983).

\section{Results}

Table 1 shows the results of Experiment 2 and reveals that the colored-strips analogue produced $95 \%$ success on the $\mathrm{x}$-ray problem and that the Red Adair analogue yielded only a $75 \%$ success rate, the difference being significant at $p<.001\left(\chi^{2}=6.38\right.$, one-tailed $)$. The summative intensity diagram was significantly less successful than were the colored strips $\left(82 \%\right.$ vs. $\left.95 \%, \chi^{2}=4.54, p<.01\right)$ and was more successful than Gick and Holyoak's (1983) diagram $\left(82 \%\right.$ vs. $\left.62 \%, \chi^{2}=3.47, p<.01\right)$.

As do those of Experiment 1, these results indicate that problem solving is facilitated by both the visual analogues that represent the summative effect and different intensities. The negligible effect of the diagram used by Gick and Holyoak (1983) was replicated. Moreover, the most effective visual analogue was the nondiagrammatic colored-strips analogue.

\section{EXPERIMENT 3}

Experiment 3 focused on the possible second function of visual representations in analogical problem solving discussed earlier. This concerns the capacity of such representations-for example, diagrams-to facilitate recall of an information structure that will lead to the solution of the problem. In Experiment 3, the x-ray problem was presented together with a diagram that represented the problem and that had previously been used in the Red Adair analogue to present the presolution condition. The diagram representing the problem was therefore common to the analogue and the problem, whereas the diagram representing the solution appeared only in the analogue. It was hypothesized that presentation of the first of the two diagrams representing the problem-solution relationship would assist recall of the solution.

\section{Method}

A total of 174 10- and 11-year-old children from six different schools were allocated to the following groups and experimental conditions. Twenty-nine children from two schools formed a control group which received the modified $x$-ray problem accompanied by the first part of Figure 2; 73 children from four schools received the Red Adair analogue accompanied by both parts of Figure 2 and then attempted to solve the modified $x$-ray problem without any diagram as a recall cue; and 72 children were given the Red Adair analogue accompanied by both parts of Figure 2 and then the modified $x$-ray problem with the first part of Figure 2. Both the second and third groups were given the analogue recall questions as in Experiment 1.

\section{Results}

The results (see Table 2) show that none of the children in the control group solved the $x$-ray problem. Of the other two groups, $12 \%$ were successful without a diagram in the problem, and $23 \%$ were successful when the diagram was present in the problem; this difference is significant at the $p<.05$ level $\left(\chi^{2}=2.39\right.$, one-tailed).

These results suggest that presenting a problem and its visual representation together facilitates recall of a solution. This occurs when a visual representation of the solution has been presented previously in association with the visual representation of the problem.

\section{EXPERIMENT 4}

As indicated in the introduction to Experiment 2, there were important differences between the presentation of the x-ray problem used by Gick and Holyoak (1983) and that used for the children in Experiments 1 and 3. Experiment 4 was essentially a replication of Experiment 3 but using the same version of both the $\mathrm{x}$-ray problem and the Red Adair analogue as in Gick and Holyoak's (1983) study. As in Experiment 2, the purpose of Experiment 4 was to determine whether the positive results of Experiment 3 could be attributed to an interaction between the diagrams and the additional information given to the children to explain the problem.

\section{Method}

Ninety-nine adult subjects, divided into two groups, participated. The 40 subjects in the first group received the Red Adair analogue with the accompanying diagrams as shown in Figure 2 and then attempted the $x$-ray problem without any diagrammatic cue. The 51 subjects in the second group received the Red Adair analogue and diagram but received the $x$-ray problem with the first part of Figure 2 as a visual recall cue.

\section{Results}

As Table 2 shows, $82 \%$ of the subjects solved the $\mathrm{x}$ ray problem after the analogue but without a cuing dia- 
Table 2

Number and Percentage of Correct Solutions to the Problem in Experiments 3 and 4

\begin{tabular}{|c|c|c|c|c|}
\hline \multirow[b]{3}{*}{ Experimental Condition } & \multicolumn{4}{|c|}{ Correct Solutions } \\
\hline & \multicolumn{2}{|c|}{ Experiment 3 (Children) } & \multicolumn{2}{|c|}{ Experiment 4 (Adults) } \\
\hline & Number & Percentage & Number & Percentage \\
\hline Control & $0 / 29$ & 0 & $23 / 40$ & 51 \\
\hline $\begin{array}{l}\text { Red Adair }+ \\
\text { Summative Intensity } \\
\text { Diagram (No diagram } \\
\text { in the problem) }\end{array}$ & $8 / 64$ & 12 & $33 / 40$ & 82 \\
\hline $\begin{array}{l}\text { As above (With } \\
\text { diagram in the problem) }\end{array}$ & $13 / 56$ & 23 & $48 / 51$ & 94 \\
\hline
\end{tabular}

gram when the problem was presented, whereas $94 \%$ solved it when the diagram was included. The difference between these two groups is significant at the $p<.02$ level $\left(\chi^{2}=3.08\right.$, one-tailed $)$. These results support those of Experiment 3.

As in Experiment 3, a visual recall cue was shown to be effective. This is further evidence of the role of visual representation in analogical problem solving.

\section{DISCUSSION}

The results of Experiments 1 and 2 indicate that Gick and Holyoak's (1983) failure to find that visual analogues were effective in problem solving was due to the nature of their diagram. Analogical reasoning depends on recognition of a structural correspondence between the analogue and the problem (Arber, 1944; Sloman, 1976, 1978). Both the diagrams in Figure 2 and the colored-strips display contain information relating to the summation of intensity at the focal area and thus correspond more precisely to the structure of the convergent solution to the $x$-ray problem. Furthermore, the analogical relationship involves more than correspondence of individual elements (Durrenberger \& Morrison, 1977; Gentner \& Gentner, 1983; Greeno, 1983; Rumelhart \& Abrahamson, 1973). In an analogue to the $x$-ray problem, the relationship among the direction, intensity, and summation is also important.

The diagrams in Figure 2 and the colored-strips display are both isomorphic (Bochenski, 1962; Evans, 1968; Gallagher, 1978) with respect to the X-ray problem solution in that this relationship is explicitly represented. The diagram used by Gick and Holyoak (1983) did not explicitly represent summation of intensity, which would lessen the degree of their diagram's correspondence to the $\mathrm{x}$-ray problem solution. This occurs in terms of the level of elemental correspondence and in terms of the overall meaning that can be attributed to those features that Gick and Holyoak's (1983) diagram does represent, that is, direction and number of radials. If intensity change is not represented, the purpose of changing the direction and number of radials is also left to inference. The results of Experiments 1 and 2 indicate that visual analogues that overcome these difficulties can be effective.

Experiments 1 and 2 also show that the modified visual analogues are more effective than the Red Adair story ana- logue. One possible reason for this difference in effectiveness is that the Red Adair story analogue contains more details that are irrelevant to the $\mathrm{x}$-ray problem. The optimum level of representation of an analogue minimizes mismatching details as well as maximizes the degree of correspondence (Gick \& Holyoak, 1983). In the Red Adair analogue, the relevant features are embedded in irrelevant information that is a necessary part of the story. In contrast, the visual analogues present the relevant information structure without any unnecessary semantic details that would then have to be seen as irrelevant to the $\mathrm{x}$-ray problem.

Gick and Holyoak's (1983) analysis of schema formation from story analogues suggested that presentation of only one story analogue does not lead to identification of a general problem solution schema: It is only when two story analogues are presented that the irrelevant mismatching details tend to be ignored and a schema formed. The relative success of the visual analogues may therefore be due to the absence of irrelevant information that would then have to be discarded before a problem-solution schema could be formed. The present study presents no direct evidence of schema formation, but the effect of our visual analogues on schema formation could be examined, as in Gick and Holyoak (1983).

Explanation of the overall superiority of the coloredstrips analogue must also be speculative. This analogue was selected for two reasons. First, it does not require an understanding of diagrammatic conventions such as arrows and shading. Second, its eye-catching quality (Willows et al., 1981) should increase its imageability and consequent recall. The effects of these different features cannot be separated on the basis of the results of the present experiments, but this will be done in future work using computer-driven visual analogues. The possible importance of imageability of the analogue in problem solving is supported by the work of Marschark and Hunt (1985) and Nall (1983) in the related area of metaphor comprehension (Gick \& Holyoak, 1983). These studies show that imageability is positively associated with comprehension and recall of metaphors. In a similar way, the imageability of visual analogues may contribute to their effectiveness.

Experiments 3 and 4 investigated the use of visual analogue recall cues in the form of a diagram. The results of Experiment 3 show that none of the children receiv- 
ing the problem with a diagram included but without a prior analogue succeeded in solving the problem. They therefore did no better than the group of children who received the problem without a diagram and without a prior analogue, as reported in Experiment 1. Therefore, inclusion of the diagram within the problem does not in itself seem to improve significantly the chances of finding the dispersion solution. Of those children receiving the Red Adair story plus the summative intensity diagram, followed by the problem without a cuing diagram, $12 \%$ succeeded in solving the problem, whereas $23 \%$ of those receiving the Red Adair story plus summative intensity diagrams, followed by the problem with a cuing diagram, succeeded. Thus, inclusion of the cuing diagram significantly improved rate of success $(p<.05)$.

Repetition of this procedure with Gick and Holyoak's (1983) problem and story version with university students in Experiment 4, which included a cuing diagram, also significantly improved success. It may therefore be concluded that a visual representation of information presented with both the problem and the analogue improves the likelihood that the analogue will be used successfully to solve the problem.

Comparison of the results for the Red Adair story versus the Red Adair story plus summative intensity diagram, for the children ( $14 \%$ vs. $12 \%$ ) and for the students ( $75 \%$ vs. $82 \%$ ), under those conditions in which no diagram was included in the problem, shows that the addition of the diagram in the analogue did not significantly improve success. This is the same result obtained by Gick and Holyoak (1983) when they added their diagram to their story. However, it must also be noted that comparison of the results for the Red Adair story plus summative intensity diagram $(82 \%)$ versus the summative intensity diagram alone ( $82 \%$ ) shows no difference. It can therefore be argued that, for this diagram, the story did not significantly improve on the effect of the visual analogue.

What is the role of the visual diagram within the problem? It does not improve success in solving the problem without an analogue; hence, the diagram does not in itself significantly add to the understanding of the problem. Because it is known that all the children in Experiment 3 whose problem answers were included had remembered the relevant points from the analogue, it may be assumed that the information was available in memory when the problem was attempted. There are two possible ways in which the diagram in the problem may be of assistance. First, and perhaps most simply, it may act as a visual recall cue, that is, a visual stimulus within the problem that matches the initial state of the visual stimulus within the analogue solution: To use Gick and Holyoak's (1983) terminology, the problem diagram can be mapped directly onto the causal antecedent of the known outcome. The problem diagram can then serve to aid recall of the second part of the diagram within the analogue along with the rest of the semantic sequence that constitutes a possible type of solution.
Alternatively, it may be that the diagram within the problem assists in freeing the relevant information from its original Red Adair context. Presenting a diagram in the problem might trigger separation of the irrelevant mismatching details presented in the Red Adair analogue by inducing more direct comparison of analogue and problem. This explanation, however, is less likely, because, as shown in Experiment 2, the summative intensity diagram on its own is an effective analogue. The subjects of Experiments 3 and 4 should therefore have developed enough understanding of the dispersion solution schema to allow the diagram in the problem to act as a recall cue.

In summary, the experiments reported here have shown that visual representation can play a part in solving spatial problems, as suggested by Chafe (1976), Kintsch (1974), Kosslyn and Pomerantz (1977), and Norman and Rumelhart (1975). Evidence has been presented for two functions of visual representations, those of visual analogue and of visual recall cue; these results were found both for adults and for children. Furthermore, the facilitative effect of the colored-strips display suggests that a quality such as "imageability" may also be important in the effect of visual analogues.

\section{REFERENCES}

ARber, A. (1944). Analogy in the history of science. In M. F. Ashley Montague (Ed.), Studies and essays in the history of science and leaming (pp. 221-223). New York: Schuman.

BECK, D. E. F. (1978). Metaphor as a mediator between semantic and analogic modes of thought. Current Anthropology, 19, 83-97.

BocHENSKI, I. M. (1962). On analogy. In I. M. Bochenski (Ed.), Logicophilosophical studies (pp. 97-117). Dordrecht, The Netherlands: Reidel.

CHAFE, W. L. (1976). Creativity in verbalization as evidence for analogic knowledge. In R. C. Shank \& B. L. Nash-Webber (Eds.), Theoretical issues in natural language processing (pp. 158-159). Cambridge, MA: Bolt, Beranek, and Newman.

DriestaDT, R. (1969). The use of analogies and incubation in obtaining insights in creative problem solving. Journal of Psychology, 71, 159-175.

DunCKer, K. (1945). On problem solving. Psychological Monographs. 58(5, Whole No. 270).

DURRENBERger, P., \& MORRISON, J. W. (1977). Theory of analogy. Journal of Anthropological Research, 33, 372-387.

EvaNs, T. G. (1968). A program for the solution of geometric-analogy intelligence test questions. In M. L. Minsky (Ed.), Semantic information processing (pp. 271-353). Cambridge, MA: M.I.T. Press.

Gallagher, J. M. (1978). The future of formal thought research: The study of analogy and metaphor. In B. Presseisen \& D. Goldstein (Eds.), Topics in cognitive development: Language and operational thought (pp. 77-98). New York: Plenum Press.

GentNER, D., \& GentNer, D. R. (1983). Flowing waters or teeming crowds: Mental models of electricity. In D. Gentner \& A. L. Stevens (Eds.), Mental models (pp. 213-226). London: Erlbaum.

Gick, M. L., \& HolYoak, K. J. (1980). Analogical problem solving. Cognitive Psychology 12, 306-355.

GICK, M. L., \& HOLYOAK, K. J. (1983). Schema induction and analogical transfer. Cognitive Psychology, 15, 1-28.

Greeno, J. G. (1983). Conceptual entities. In D. Gentner \& A. L. Stevens (Eds.), Mental models (pp. 227-252). Hillsdale, NJ: Erlbaum. Holyoak, K. J., JunN, E., \& Billman, D. O. (1984). Development 
of analogical problem solving skill. Child Development, 55, 2042-2055.

$\mathrm{KINTSCH}, \mathrm{W}$. (1974). The representation of meaning in memory. Hillsdale, NJ: Erlbaum.

KossLYN, S. M. (1975). Information representation in visual images. Cognitive Psychology, 7, 341-370.

Kosslyn, S. M., Pomerantz, J. R. (1977). Imagery, propositions and the form of internal representations. Cognitive Psychology, 9, 52-76.

Marschark, M., \& HUNT, R. R. (1985). On memory for metaphor. Memory \& Cognition, 13, 413-424.

NALL, L. (1983). Dimensions of metaphor comprehensionin third, fift and seventh graders. Unpublished master's thesis, Wake Forest University, Winston-Salem, NC.

Norman, D. A., \& Rumelhart, D. E. (1975). Memory and knowledge. In D. A. Norman \& D. E. Rumelhart (Eds.), Explorations in cognition (pp. 3-32). San Francisco: Freeman.

Palmer, S. E. (1976). The nature of perceptual representation: An examination of the analog/propositional controversy. In R. C. Schank \& B. L. Nash-Webber (Eds.), Theoretical issues in natural language processing (pp. 165-172). Cambridge, MA: Bolt, Beranek, and Newman.
ReEd, S. K., ERnst, G. W., \& BANERI, R. (1974). The role of analogy in transfer between similar problem states. Cognitive Psychology 6, 436-450.

Rumelhart, D. E., \& Abrahamson, A. A. (1973). A model for analogical reasoning. Cognitive Psychology, 5, 1-28.

SHEPARD, R. N. (1975). Form, formation and transformation of internal representations. In $\mathrm{R}$. L. Solso (Ed.), Information processing and cognition (pp. 87-122). Hillsdale, NJ: Erlbaum.

Sloman, A. (1976). Afterthought on analogical representation. In R. C Schank \& B. L. Nash-Webber (Eds.), Theoretical issues in natural language processing (pp. 107-110). Cambridge, MA: Bolt, Beranek, and Newman.

SLoman, A. (1978). The computer revolution in philosophy: Philosophy, science, and models of mind. Sussex: Harvester.

Willows, D. M., Borwick, D., \& Hayvren, M. (1981). The content of school readers. In G. E. Mackinnon \& T. G. Waller (Eds.), Reading research: Advances in theory and practice (pp. 100-177). London: Academic Press.

(Manuscript received October 11, 1985; revision accepted for publication September 24, 1986.) 